\title{
Collective excitations of an laser driven atomic condensate in an optical cavity
}

\author{
B. Öztop ${ }^{1}$ Ö. E. Müstecaploğlu, ${ }^{2, *}$ and H. E. Türeci ${ }^{3,1}$ \\ ${ }^{1}$ Institute for Quantum Electronics, ETH-Zürich, CH-8093 Zürich, Switzerland \\ ${ }^{2}$ Department of Physics, Koç University, Istanbul, 34450, Turkey \\ ${ }^{3}$ Department of Electrical Engineering, Princeton University, Princeton, New Jersey 08544, USA
}

(Dated: November 1, 2011)

\begin{abstract}
We theoretically examine collective excitations of an optically driven atomic Bose-Einstein condensate, coupled to a high finesse optical cavity. This open system has been recently used for the experimental demonstration of Dicke superradiance of cavity photons, which is simultaneously and mutually triggered by spontaneous breaking of translational symmetry of the condensate into a crystalline order. We first develop Hartree-Fock mean field dynamical model of the physical system. Using this model, we compute the dynamics of the cavity photons, the condensate density profile and the Dicke phase transition diagram. Both the imaginary time and real time evolution methods are used in the calculations. Collective excitations are determined by the solving Bogoluibov- de Gennes equations. Spectrum, softening of the modes and energetic hierarchy of excitations are determined.
\end{abstract}

Topic: Physics of Cold Trapped Atoms (Report number: 6.3.1)

PACS numbers: 03.75.Kk / 03.75.Lm / 37.10.Vz

\section{INTRODUCTION}

Elementary excitations of Bose-Einstein condensates (BECs) have attracted much interest in harmonic traps 1 and in optical lattices 2. Studies on nonlinear instabilities, solitons and other topological excitations are not only of fundamental curiosity, but they also have potential impact on matter-wave and quantum information applications [3]. Controlled generation and detection of collective modes, as well as means of enhancing or suppressing them, by field gradient, tilted or modulated traps, and by Bragg spectroscopy methods are actively sought for such practical problems [4] 8. In addition, such investigations are necessary for explorations of dynamics of quantum phase transitions, where it is known that strong collective fluctuations and correlations emerge at the transition points.

Predicted self-organized quantum phase transitions of a BEC coupled to a driven high-finesse optical cavity [9] has been recently demonstrated experimentally, albeit in a two-dimensional system [10. More recently, symmetry breaking between two degenerate self-organized motional states of the condensate at the Dicke phase transition has been observed in real-time monitoring by optical heterodyne method [11. Spectrum of low-lying collective excitations in such a system has been investigated using a one-dimensional model [12 under mean-field approximation to a quantum mechanical system 13 .

The system goes through a self-organization transition from a homogeneous Bose-gas to a crystalline structure at a certain threshold of drive strength. Bragg scattering of the driving laser off the BEC into the cavity field and the emergence of the crystal lattice mutually enhances each other. Structural transformation of the atomic subsystem is accompanied by a simultaneous Dicke phase transition (for a recent review see Ref. [14) of the cavity field from vacuum state to a superradiant state. Theoretical investigations examine critical exponents and rich phase diagrams associated with these systems, not fully explored in the experiments, or found in the usual Dicke model [15, 18]. Multimode cavity field effects on phase transitions have been analysed recently 19, 20. The crucial difference of this system, from the point of view of collective modes of trapped condensates, is the polaritonic structure of the excitations, which are coupled modes of fluctuations both of the cavity field and the condensate.

While cavity mediated long range interactions between the atoms would lead to similar self-organized crystalline phases also in thermal gases [21, superfluid coherent character of the atomic condensates allow for novel phases, as supersolidity 22 24, and distinct collective excitations, as Bogoluibov quasiparticles.

In this contribution, we consider the actual two-dimensional geometry of the recent experiments [10, 11] and examine the collective excitations. For that aim, we first reproduce the experimental results theoretically using Hartree-Fock mean field approach. Dynamical evolutions of the cavity field and the condensate density profile depending on the drive

*Electronic address: omustecap@ku.edu.tr 
strength and cavity-pump detuning are obtained by solving the coupled mean field equations in real time using splitstep Fourier numerical computations. In addition, stationary states are analysed using imaginary time propagation numerical methods. Phase diagram of the Dicke quantum phase transition is recovered by this a treatment. Combining these results with the Bogoliubov-de Gennes analysis, we obtain the spectrum, dynamics and spatial profile of the excitations. We discuss softening of the collective modes and present an energetic hierarchy of excitations.

The organization of the paper is as follows. Our physical system is described in Sec. II], followed by the mean field model of it in Sec. III. In Sec. IV], dynamics of collective excitations are investigated. Finally we conclude in Sec. V]

\section{PHYSICAL SYSTEM}

We consider an atomic Bose-Einstein condensate of $N$ two level atoms inside a high finesse optical cavity. The atoms are additionally kept in a far off-resonant dipole trap (FORT). The condensate is pumped by a laser transverse to the cavity axis. If the laser is sufficiently large detuned from the atomic transition frequency, the excited states of the atomic system can be eliminated from the dynamics adiabatically. Starting from the Jaynes-Cummings model for the interaction of a single atom with the cavity mode, under electric-dipole and rotating-wave approximations, and taking into account external and internal degrees of freedom of the atom, an effective many-body Hamiltonian description of the physical system is obtained by employing the standard second quantization procedure [13, such that

$$
\begin{aligned}
& H=\int \mathrm{d}^{3} \mathbf{x} \Psi^{\dagger}(\mathbf{x})\left\{T+V(\mathbf{x})+\frac{U}{2} \Psi^{\dagger}(\mathbf{x}) \Psi(\mathbf{x})\right. \\
& \left.+\frac{\hbar}{\Delta_{a}}\left[h^{2}(\mathbf{x})+g^{2}(\mathbf{x}) \hat{a}^{\dagger} \hat{a}+h(\mathbf{x}) g(\mathbf{x})\left(\hat{a}+\hat{a}^{\dagger}\right)\right]\right\} \Psi(\mathbf{x}) \\
& -\hbar \Delta_{c} \hat{a}^{\dagger} \hat{a}
\end{aligned}
$$

where $T=-\left(\hbar^{2} / 2 m\right) \nabla^{2}$ is the kinetic energy operator for atoms of mass $m, V(\mathbf{x})$ is the FORT potential for the atoms in the ground state, $\Psi(\mathbf{x})$ is the Schrödinger field operator that annihilates an atom at position $\mathbf{x}$ in the ground state. The strength of the two-atom collision is characterized by $U=4 \pi \hbar^{2} a_{s} / m$, with $a_{s}$ being the s-wave scattering length. Mode-functions of the pump and cavity fields are respectively denoted by $h(\mathbf{x})$ and $g(\mathbf{x})$. Cavity photon annihilation operator is denoted by $\hat{a}$. Detuning of the cavity mode frequency $\omega_{c}$ from the frequency of the pump $\omega_{p}$ is $\Delta_{c}=\omega_{p}-\omega_{c}$. Pump laser is detuned from the atomic transition frequency by $\Delta_{a}$.

In parallel with the recent experiments, we consider the case in which the trap potential is deep in the $z-$ direction, transverse both to the cavity axis (x) and the pump axis (y). Accordingly, rescaling the two-body interaction strength to $U_{2 D}=(8 \pi)^{(1 / 2)} \hbar^{2} a_{s} /\left(m a_{z}\right)$, with $a_{z}$ being the size of the harmonic oscillator ground state along the suppressed direction $z$, we can treat the model effectively as a $2 \mathrm{D}$ system, and choose the coordinate frame such that $\mathbf{x}=(x, y)$.

\section{HARTREE-FOCK MEAN FIELD MODEL OF THE SYSTEM}

In the mean field approximation we replace the quantum operators by classical numbers (c-numbers) in the Heisenberg equations of motions, which become

$$
\begin{gathered}
\frac{\partial \psi(x, y ; t)}{\partial t}=-\mathrm{i}\left\{-\frac{\hbar}{2 m}\left(\frac{\partial^{2}}{\partial_{x}^{2}}+\frac{\partial^{2}}{\partial_{y}^{2}}\right)+\frac{V_{2 D}(x, y)}{\hbar}\right. \\
+\frac{U_{2 D}}{\hbar}|\psi(x, y ; t)|^{2}+\frac{\Omega_{p}^{2}}{\Delta_{a}} \phi_{p}^{2}(x, y)+\frac{g_{0}^{2}}{\Delta_{a}} \phi_{c}^{2}(x, y)|\alpha(t)|^{2} \\
\left.+2 \eta \phi_{p}(x, y) \phi_{c}(x, y) \operatorname{Re}[\alpha(t)]\right\} \psi(x, y ; t), \\
\frac{\partial \alpha(t)}{\partial t}=\left[-\mathrm{i} \frac{g_{0}^{2}}{\Delta_{a}} B(t)+\mathrm{i} \Delta_{c}-\kappa\right] \alpha(t)-\mathrm{i} \eta \Theta(t),
\end{gathered}
$$

where the two-dimensional trap potential is taken to be $V_{2 D}(x, y)=m\left(\omega_{x}^{2} x^{2}+\omega_{y}^{2} y^{2}\right) / 2$. The pump and cavity mode functions $h(x, y)$ and $g(x, y)$ are respectively scaled by the corresponding Rabi frequencies $\Omega_{p}$ and $g_{0}$. We take $\phi_{p}=h(x, y) / \Omega_{p}=\cos \left(k_{p} y\right) \exp \left(-x^{2} / w_{p}^{2}\right)$ and $\phi_{c}=g(x, y) / g_{0}=\cos \left(k_{c} x\right) \exp \left(-y^{2} / w_{c}^{2}\right)$ with $w_{p}$ and $w_{c}$ being the pump laser beam waist and waist radius of the cavity $\mathrm{TEM}_{00}$ mode, respectively. The wave numbers for the cavity and the pump modes are $k_{c}$ and $k_{p}$, respectively. We introduce an effective two-photon Rabi frequency as $\eta=\Omega_{p} g_{0} / \Delta_{a} . \mathrm{A}$ so called bunching parameter $B(t)=\left\langle\psi\left|\phi_{c}^{2}(x, y)\right| \psi\right\rangle$ and order parameter $\Theta(t)=\left\langle\psi\left|\phi_{p}(x, y) \phi_{c}(x, y)\right| \psi\right\rangle$ are defined. 
Loss rate of the cavity photons is determined by a phenomenological constant $\kappa$. The equations are subject to constraint by the normalization condition $\int \mathrm{d}^{2} \mathbf{x}|\psi(x, y ; t)|^{2}=N$. This system of equations is a generalization of the $1 \mathrm{D}$ case treated in Ref. 12 .

The system of mean field equations can be more conveniently investigated by using scaled field and dimensionless space-time variables. For that aim we consider a characteristic time scale as the recoil frequency $\omega_{R}=\hbar k^{2} / 2 m$, with $k$ being the recoil wave number, and a characteristic length scale as the recoil wavelength of atoms, $\lambda=2 \pi / k$. We consider the perfectly phase matched case so that $k_{p}=k_{c}=k$. For the field variables the scaling is by the atom number such that $\psi \rightarrow \lambda \psi / \sqrt{N}$ and $\alpha \rightarrow \alpha / \sqrt{N}$. The normalization condition of the scaled matter field in the dimensionless space-time now becomes $\int \mathrm{d}^{2} \mathbf{x}|\psi(x, y ; t)|^{2}=1$. The equation system becomes

$$
\begin{aligned}
& \mathrm{i} \frac{\partial \psi(x, y ; t)}{\partial t}=\left\{-\frac{1}{4 \pi^{2}}\left(\frac{\partial^{2}}{\partial_{x}^{2}}+\frac{\partial^{2}}{\partial_{y}^{2}}\right)+V_{2 D}(x, y)\right. \\
+ & U_{2 D}|\psi(x, y ; t)|^{2}+U_{p} \phi_{p}^{2}(x, y)+U_{0} \phi_{c}^{2}(x, y)|\alpha(t)|^{2} \\
+ & \left.2 \eta \phi_{p}(x, y) \phi_{c}(x, y) \operatorname{Re}[\alpha(t)]\right\} \psi(x, y ; t), \\
& \mathrm{i} \frac{\partial \alpha(t)}{\partial t}=\left[U_{0} B(t)-\Delta_{c}-\mathrm{i} \kappa\right] \alpha(t)+\eta \Theta(t),
\end{aligned}
$$

where all the frequencies and energies are scaled by the recoil frequency and the recoil energy $E_{R}=\hbar \omega_{R}$. Under the scaling transformations we have $U_{2 D} \rightarrow N U_{2 D} / \lambda^{2}$ and $\eta \rightarrow \sqrt{N} \eta$. We defined $U_{0}=N g_{0}^{2} / \Delta_{a}$ and $U_{p}=\Omega_{p}^{2} / \Delta_{a}$.

In contrast to imaginary time evolution method, which is typically used to examine steady state solutions of such coupled system of nonlinear Schrödinger equations, numerical analysis of real time evolution of the system faces serious challenge of numerical instabilities. We employ a second-order pseudospectral, symmetric Strang split-step algorithm to determine numerical solution of the system in real time domain [25]. This algorithm is a popular symplectic integration method. It has been recently shown that the latent numerical instabilities of the splitting process, associated with the noises of Bogoluibov elementary excitations, can be avoided provided that $\pi \Delta t / \Delta x^{2} \lesssim 2$, where $\Delta x$ and $\Delta t$ are the spatial and temporal step sizes, respectively [26]. Splitting of the kinetic and potential energy integrators with their exact solutions is only used for the condensate mean field equation. The coupled cavity field equation is evolved by standard finite differencing in time domain.

In our typical simulations we use the parameters for the experimental system of Ref. [10] where $N=1.5 \times 10^{5} 87 \mathrm{Rb}$ atoms, $a_{s}=5.1 \mathrm{~nm}, m=1.44 \times 10^{-25} \mathrm{~kg}, \omega_{x} / 2 \pi=252 \mathrm{~Hz}, \omega_{y} / 2 \pi=238 \mathrm{~Hz}, w_{c}=25 \mu \mathrm{m}, w_{p}=29 \mu \mathrm{m}, \lambda=784.5$ $\mathrm{nm}, \kappa / 2 \pi=1.3 \mathrm{MHz}, \Delta_{a}=-4.3 \mathrm{~nm}, \Delta_{c} / 2 \pi=-14.9 \mathrm{MHz}, g_{0} / 2 \pi=10.4 \mathrm{MHz}$. We calculate $\omega_{R} / 2 \pi \sim 3.72$ $\mathrm{kHz}$. Following the experiment, we consider the influence of several control parameters, in particular different cavity detunings $\Delta_{c}$, and variations of pump power, by changing $\Omega_{p}$. The effects of such control parameters can be followed by physical parameters of $\eta, U_{p}$ and $U_{0}$. Typically $\Omega_{p}$ and $\Delta_{a}$ are chosen to produce a lattice depth of $U_{p} \sim 10 E_{R}$. The length of the condensate is reported to be about $\sim 30 \lambda$. This makes about $10^{4}$ atoms per lattice site. Neglecting any quantum correlations between cavity photons and the condensate atoms and assuming uncorrelated coherent states at all times for both, mean field description of the system is suitable both for the homogeneous and for the crystalline phases of the condensate, and even for small number of photons. To compensate lack of quantum fluctuations that would trigger spontaneous breaking of the condensate translational symmetry and associated superradiance, we introduce some seeding noise in the initial field functions.

In most of our simulations we found good agreement between the real and imaginary time evolution methods. A typical simulation result is shown in Fig. 1. In some cases however the agreement is poor as examplified in Fig. 2 . Using the imaginary time evolution method we calculate the steady state values of the photon number and plotted it in Fig. 3. This coincides with the experimentally determined and theoretically predicted Dicke superradiance phase diagram. The regimes in which the imaginary and real time evolutions shows larger deviations correspond to the upper phase boundary. This is where the instability of the system is strongest. When we examine the evolution of the order parameter we find that the system evolves in coherent superposition of two stationary and degenerate states as even and odd lattices [10. These lattices correspond to atoms occupying either even or odd numbered sites, separated by half wavelength of the pump laser, in a square lattice configuration, analogous to a checkerboard pattern [10]. This is illustrated in Fig. 4 where the order parameter and the density profile of the condensate oscillates between even and odd lattice configurations as shown in the insets. Dicke superradiance and associated structural phase transition of the condensate from homogeneous to crystalline phase by breaking of the translational symmetry is illustrated in the Fig. 5 . 


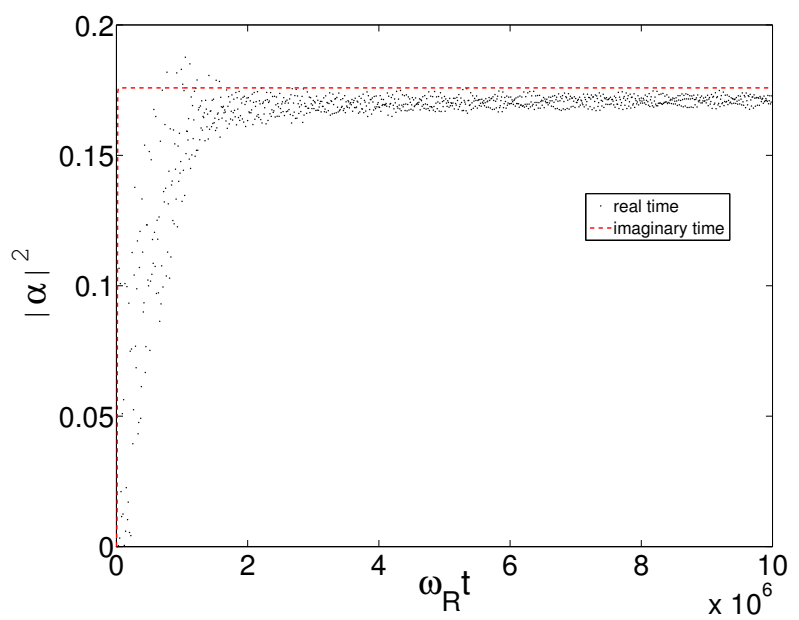

FIG. 1: (Color online) The agreement between the imaginary (black dotted curve) and real time (red dashed line) evolution methods for $\eta=100, \Delta_{c}=-100, U_{0}=-100$. Threshold for Dicke superradiant phase transition is numerically determined to be $\eta_{c}=94.5$. The scaled dimensionless parameters are used as explained in the text.

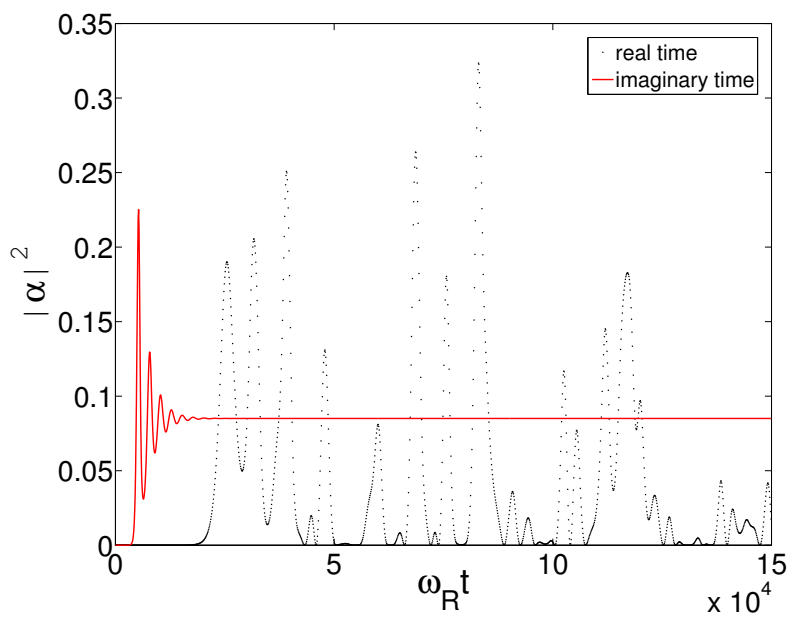

FIG. 2: (Color online) The agreement between the imaginary (black dotted curve) and real time (red dashed line) evolution methods for $\eta=200, \Delta_{c}=-70, U_{0}=-100$. Threshold for Dicke superradiant phase transition is numerically determined to be $\eta_{c}=145.6$. The scaled dimensionless parameters are used as explained in the text.

\section{COLLECTIVE EXCITATIONS}

Stationary solutions of the mean field equations can be considered as background fields out of which the system can be collectively excited (For a recent review see Ref. 27]). Denoting the steady state fields by $\psi_{0}(x, y)$ and $\alpha_{0}$, we write

$$
\begin{aligned}
\alpha(t) & =\alpha_{0}+\delta \alpha(t), \\
\psi(x, y, ; t) & =\mathrm{e}^{-\mu t}\left[\psi_{0}(x, y)+\delta \psi(x, y ; t)\right],
\end{aligned}
$$

where $\mu$ is the eigenenergy of the stationary state. If we insert these expressions into the mean field equations of motion, Eqs. 4.5, and linearize in relatively small fluctuations of $\delta \alpha$ and $\delta \psi$, we get a system of Bogoluibov-de Gennes 


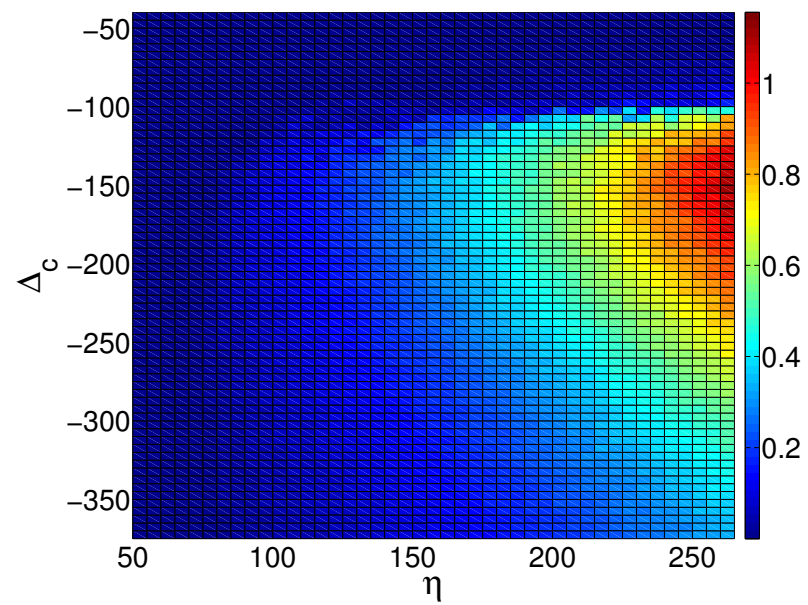

FIG. 3: (Color online) Phase diagram of the Dicke superradiance is obtained by calculating the number of photons in the cavity. The scaled dimensionless parameters are used as explained in the text.

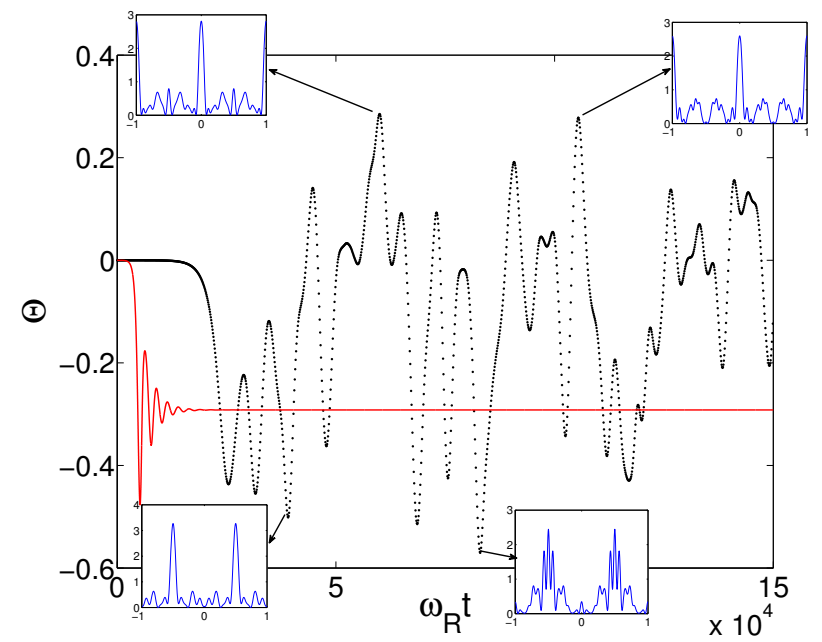

FIG. 4: (Color online) Time dependence of the order parameter for the parameters corresponding to the upper boundary of the phase diagram in Fig. 3 The insets shows the density profile of the condensate. The scaled dimensionless parameters are used as explained in the text.

equations 28

$$
\begin{aligned}
& \mathrm{i} \delta \dot{\alpha}=A \delta \alpha+\alpha_{0}\left(\left\langle\psi_{0}\left|U_{0}(x, y)\right| \delta \psi\right\rangle+c . c\right) \\
& \quad+\quad\left(\left\langle\psi_{0}|\eta(x, y)| \delta \psi\right\rangle+c . c .\right) \\
& \mathrm{i} \delta \dot{\psi}=\left(H_{0}+U_{2 D}\left|\psi_{0}\right|^{2}\right) \delta \psi+U_{2 D} \psi_{0}^{2} \delta \psi^{*} \\
& \quad+\psi_{0} U_{0}(x, y)\left(\alpha_{0} \delta \alpha^{*}+c . c .\right)+2 \psi_{0} \eta(x, y) \operatorname{Re}(\delta \alpha)
\end{aligned}
$$

where the functions $U_{0}(x, y)=U_{0} \phi_{c}^{2}(x, y)$ and $\eta(x, y)=\eta \phi_{p}(x, y) \phi_{c}(x, y)$ are introduced for notational simplicity. We also define

$$
A=-\Delta_{c}+\left\langle\psi_{0}\left|U_{0}(x, y)\right| \psi_{0}\right\rangle-\mathrm{i} \kappa,
$$



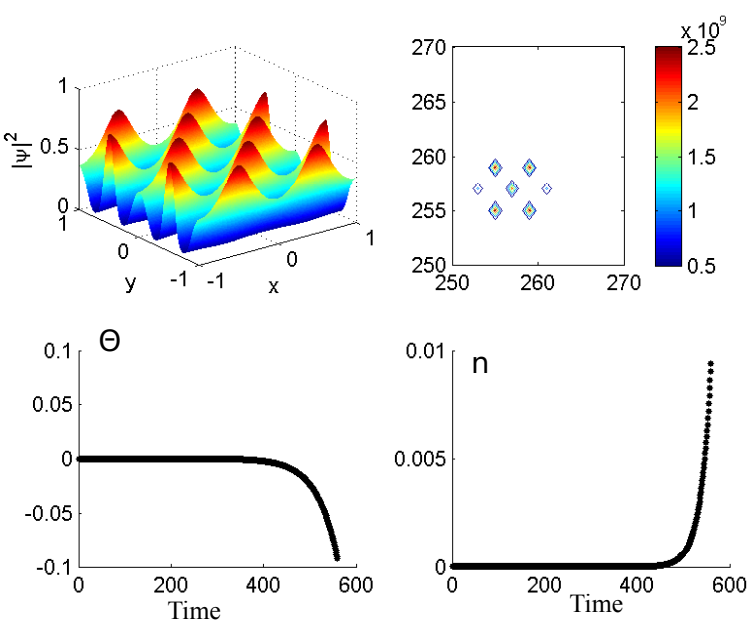

FIG. 5: (Color online) Just before the self organization of the condensate atoms and Dicke superradiance of the cavity photons, the density profile of the condensate (a), momentum space representation of the density profile (b), order parameter $\Theta$ (c), and the cavity mode population (denoted by $n$ ) (d) are shown. The scaled dimensionless parameters are used as explained in the text.

and

$$
\begin{aligned}
& H_{0}=-\frac{1}{4 \pi^{2}}\left(\frac{\partial^{2}}{\partial_{x}^{2}}+\frac{\partial^{2}}{\partial_{y}^{2}}\right)+\pi^{2}\left(\omega_{x}^{2} x^{2}+\omega_{y}^{2} y^{2}\right) \\
& +\quad U_{p} \phi_{p}^{2}(x, y)+\left|\alpha_{0}\right|^{2} U(x, y)+2 \operatorname{Re}\left(\alpha_{0}\right) \eta(x, y) \\
& +\quad U_{2 D}\left|\psi_{0}(x, y)\right|^{2}-\mu
\end{aligned}
$$

We first determine the eigenenergy spectrum of the collective excitations by writing the equation system in matrix form. For that aim we use a normal mode parametrization of Bogoliubov fluctuations such that

$$
\begin{aligned}
& \delta \alpha(t)=\delta \alpha_{+}(t) \mathrm{e}^{-\mathrm{i} \lambda t}+\delta \alpha_{-}^{*}(t) \mathrm{e}^{\mathrm{i} \lambda^{*} t} \\
& \delta \psi(t)=\delta \psi_{+}(t) \mathrm{e}^{-\mathrm{i} \lambda t}+\delta \psi_{-}^{*}(t) \mathrm{e}^{\mathrm{i} \lambda^{*} t}
\end{aligned}
$$

In our numerical simulations, $\alpha_{0}, \psi_{0}$ and $\mu$ is determined by running an imaginary time algorithm for sufficiently long times to ensure that the steady-state is reached for different parameter values. We then solve the eigensystem to find the complex eigenvalues $\lambda$ and eigenvectors $\left(\delta \psi_{+}(x, y), \delta \psi_{-}(x, y), \delta \alpha_{+}, \delta \alpha_{-}\right)$.

Real and imaginary parts of the eigenvalues $\lambda$ are plotted as a function of $\eta$ in Fig. 6. We assume condensate sizes are $3 \lambda \times 3 \lambda$ in $x, y$ directions. Negative imaginary parts of the eigenvalues in general indicate damped relaxation oscillations, i.e. decay of the mode that can be tracked by the cavity photons leaking out of the cavity. Beyond the threshold of phase transition, there are positive and negative imaginary parts of $\lambda$ for the soft modes with vanishing real parts of their eigenvalues. This is a typical signature of instability of the system. At the onset of the transition, existence of a narrow window of $\eta$ is where the imaginary part of the eigenvalue is negative while its real part is zero is associated with the dissipative cavity cooling effect 12 . The softening of some collective modes with the real part of their eigenvalues goes to zero as $\eta$ approaches to the critical value for the Dicke phase transition. In contrast to one-dimensional condensate, where single soft mode is found [12, there are several soft modes in the two-dimensional system. In order to compare the same condensate size situations and clarify the effect of dimensionality for the one and two-dimensional cases, we consider a $\lambda$ sized condensate as shown in Fig. 7 in which two modes get soft. In these figures only the first 9 eigenvalues are plotted. There are optically accessible modes in higher excitations, though they are not soft modes. Next 9 eigenvalues are shown in Fig. 8 .

Spatial profiles of the collective modes can be classified as a hierarchy of mulipole excitations. Below the Dicke phase transition threshold, the excitations exhibit typical Bogoluibov mode profiles as indicated in Fig. 9 where $\eta=30$ is taken. Above the threshold of phase transition, for example at $\eta=150$, the hierarchy of excitations are shown in Figs. 10 11. In the hierarchy of collective modes, multipole excitations along the cavity axis $x$ are less energetic and hence precede those along the pump axis. This is due to the asymmetric optical potential formed in the $x y$-plane. The potential wells along $x$-direction are wider than those along $y$-direction. This fact is reflected in Figs. 12(a) 12(b)] 

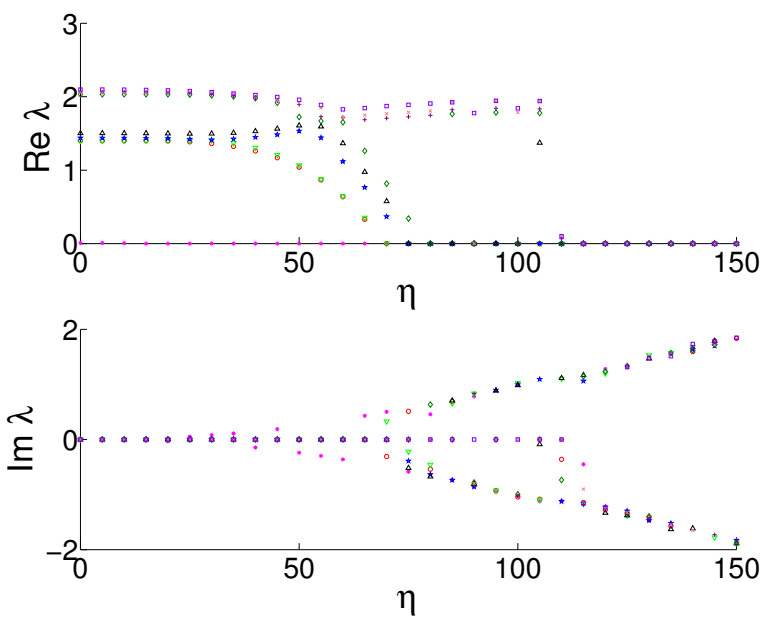

FIG. 6: (Color online) The real and imaginary parts of the eigenvalues $\lambda$ of the collective excitations for the case of a condensate of size $3 \lambda \times 3 \lambda$. The scaled dimensionless parameters are used as explained in the text.
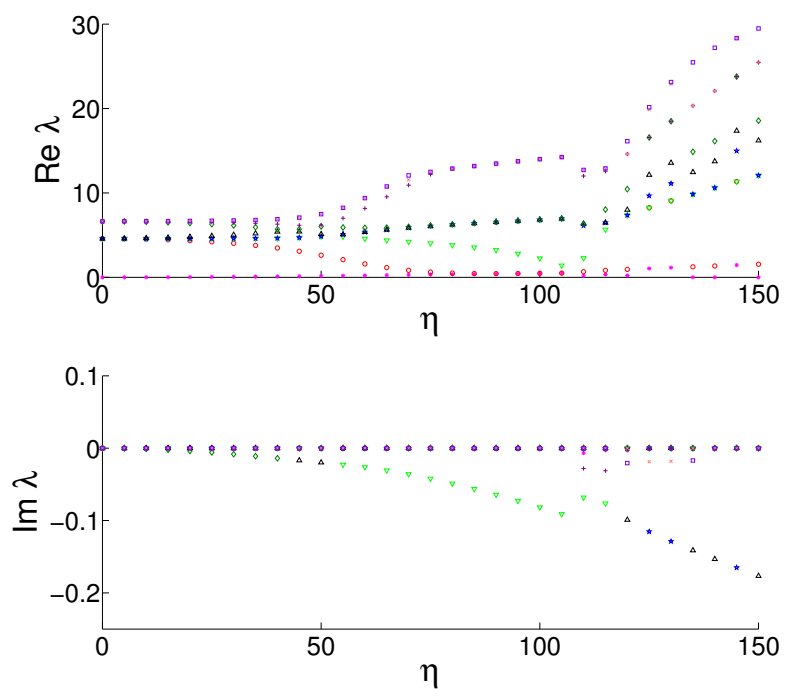

FIG. 7: (Color online) Same with Fig. 6 but for a condensate of size $\lambda \times \lambda$. The scaled dimensionless parameters are used as explained in the text.

where we plotted the optical potential

$$
\begin{aligned}
V(x, y) / \hbar & =\frac{\Omega_{p}^{2}}{\Delta_{a}} \phi_{p}^{2}(x, y)+\frac{g_{0}^{2}}{\Delta_{a}} \phi_{c}^{2}(x, y)\left|\alpha_{0}\right|^{2} \\
& +2 \eta \phi_{p}(x, y) \phi_{c}(x, y) \operatorname{Re}\left[\alpha_{0}\right]
\end{aligned}
$$

seen by the atoms for below and above threshold respectively. In addition to longitudinal and transverse excitations, lateral multipoles do emerge in the two-dimensional geometry.

\section{CONCLUSION}

In this work, we developed a mean field description of an optically driven atomic condensate, coupled to a single mode of a high-quality cavity. The condensate goes through a self-organization transition as a function of the driving strength, simultaneously with the optical cavity field undergoing Dicke superradiance phase transition. We show that 

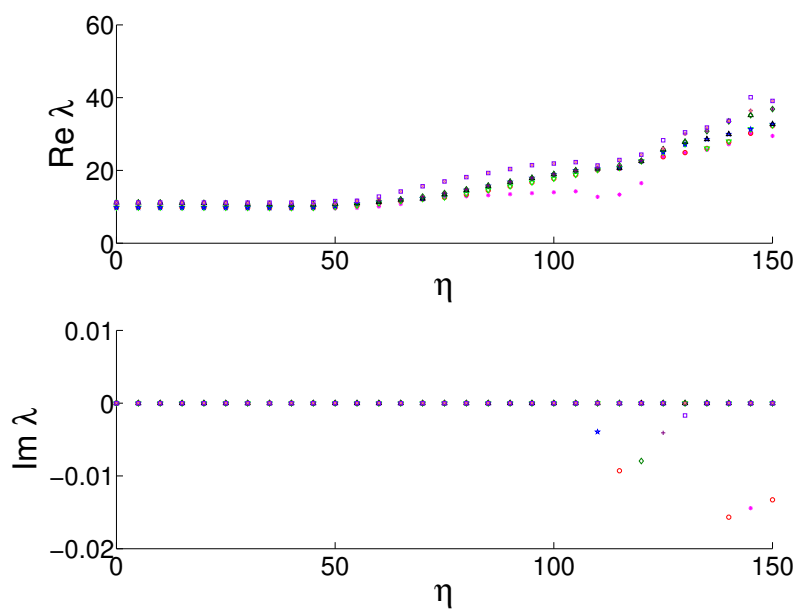

FIG. 8: (Color online) Same with Fig. 7 but for the next 9 eigenvalues. The scaled dimensionless parameters are used as explained in the text.
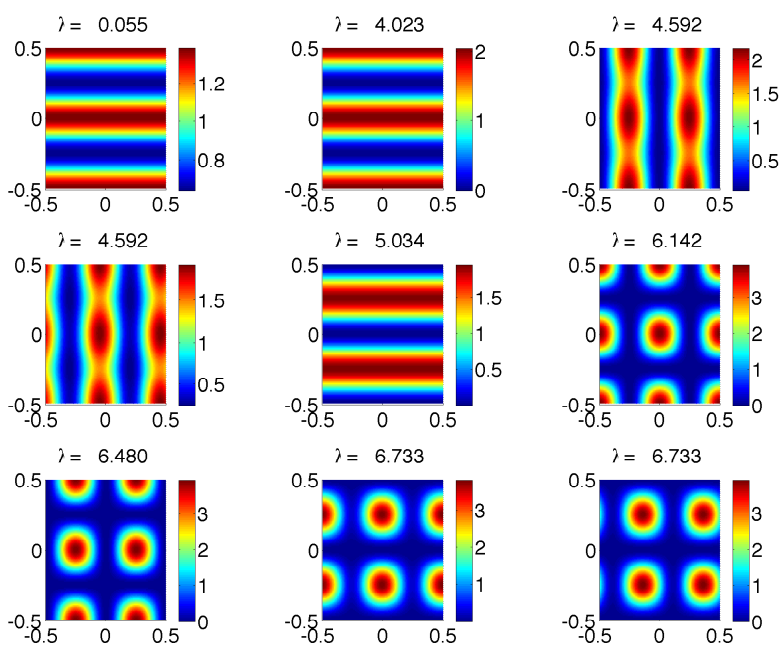

FIG. 9: (Color online) Spatial profiles of the Bogouibov modes, $\left|\delta \psi_{+}(x, y)\right|^{2}$, that are excited below the Dicke phase transition threshold at $\eta=30$. The scaled dimensionless parameters are used as explained in the text. The horizontal

Hartree-Fock mean field approach successfully reproduces the experimentally observed cavity field and condensate density profile dynamics, as well as the Dicke superradiance phase diagram. By taking into account the fluctuations out of the mean field background, we derive the Bogoluibov - de Gennes equations. Their solutions yield a hierarchy of excitations in the usual of order of multipoles. Excitations unique to two-dimensional geometry, such as lateral multipoles, are found in addition to longitudinal and transverse multipoles. Due to anisotropic effective optical trap potential, superimposed onto the magnetic trap, excitations along the cavity axis precede those transverse to the cavity axis.

\section{Acknowledgments}

The authors thank K. Baumann, F. Brennecke, R. Mottl, T. Esslinger, S. Schmidt, S. Shinohara, Y. E. Lozovik and I. B. Mekhov for stimulating discussions. H.E.T. acknowledges support from Swiss NSF under Grant No. PP00P2- 

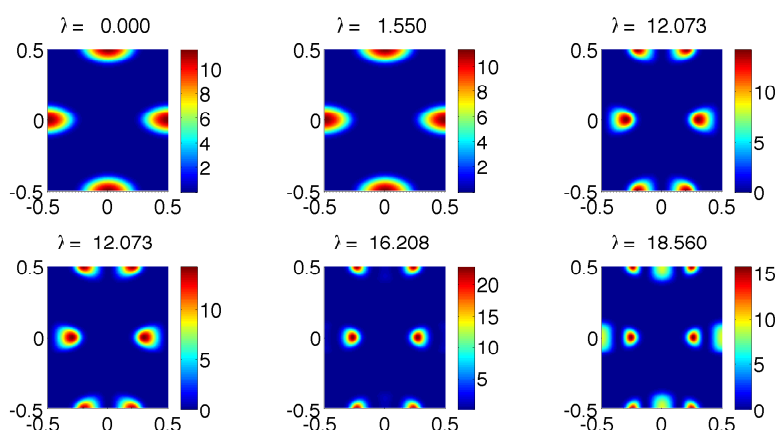

$\lambda=16.208$

$\lambda=18.560$
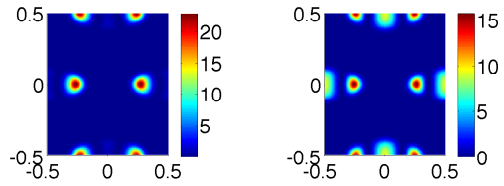

$\lambda=25.455$

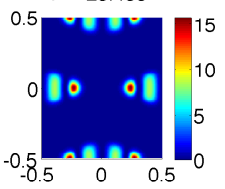

$\lambda=25.455$
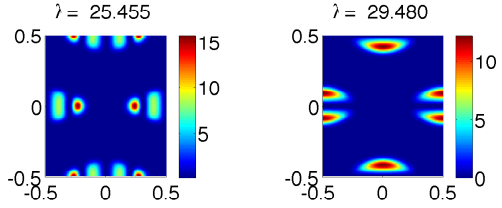

FIG. 10: (Color online) Spatial profiles of the collective modes, $\left|\delta \psi_{+}(x, y)\right|^{2}$, that are excited above the Dicke phase transition threshold at $\eta=150$. The scaled dimensionless parameters are used as explained in the text.
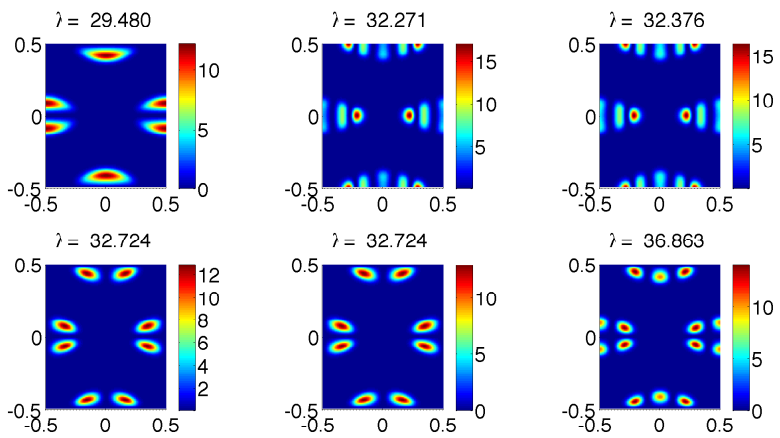

$\lambda=32.724$
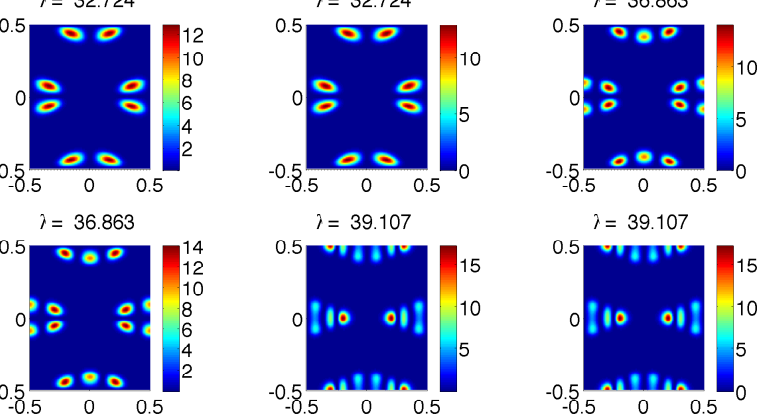

FIG. 11: (Color online) Spatial profiles of the collective modes, $\left|\delta \psi_{+}(x, y)\right|^{2}$, that are excited above the Dicke phase transition threshold at $\eta=150$. The scaled dimensionless parameters are used as explained in the text.

123519/1. Ö.E.M. acknowledges support by TUBITAK for the Project No. 109T267.

[1] F. Dalfovo, S. Giorgini, L. P. Pitaevskii, and S. Stringari, Rev. Mod. Phys. 71, 463 (1999).

[2] V. I. Yukalov, Laser Phys. 19, 1 (2009).

[3] O. Morsch and M. Oberthaler, Rev. Mod. Phys. 78, 179 (2006).

[4] M. Greiner, O. Mandel, T. Esslinger, T. W. Hansch, and I. Bloch, Nature 415, 39 (2002).

[5] C. Schori, T. Stöferle, H. Moritz, M. Köhl, and T. Esslinger, Phys. Rev. Lett. 93, 240402 (2004).

[6] J. Plata, Phys. Rev. A 69, 033604 (2004)

[7] R. Ozeri, N. Katz, J. Steinhauer, and N. Davidson, Rev. Mod. Phys. 77, 187 (2005).

[8] X. Du, S. Wan, E. Yesilada, C. Ryu, D. J. Heinzen, Z. Liang, and B. Wu, New Jour. Phys. 12, 083025 (2010). 


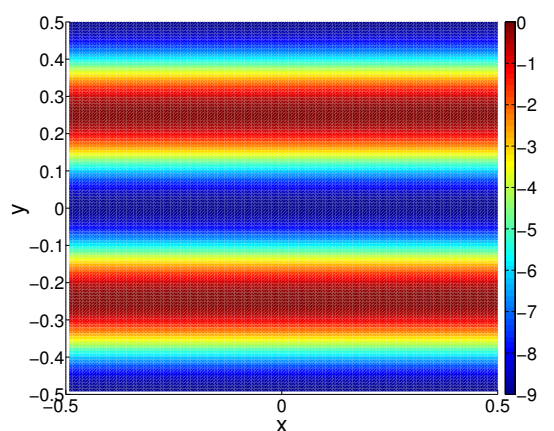

(a)

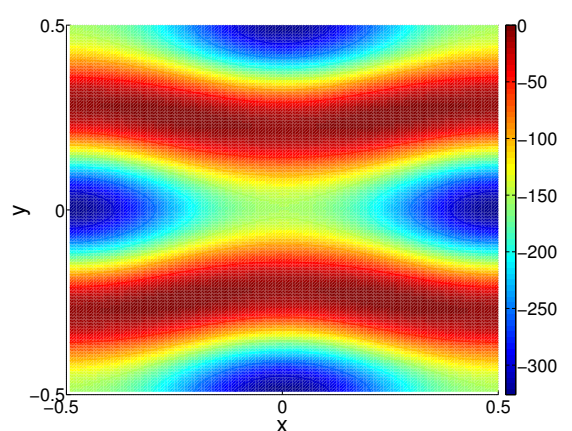

(b)

FIG. 12: Optical potential $V(x, y)$ seen by the atoms (a) below threshold for $\eta=30$ and (b) above threshold for $\eta=150$. The scaled dimensionless parameters are used as explained in the text.

[9] P. Domokos and H. Ritsch, Phys. Rev. Lett. 89, 253003 (2002).

[10] K. Baumann, C. Guerlin, F. Brennecke, and T. Esslinger, Nature 464, 1301 (2010).

[11] K. Baumann, R. Mottl, F. Brennecke, and T. Esslinger, Phys. Rev. Lett. 107, 140402 (2011).

[12] D. Nagy, G. Szirmai, and P. Domokos, Eur. Phys. J. D 48, 127 (2008).

[13] Maschler, C., Mekhov, I. B., and Ritsch, H., Eur. Phys. J. D 46, 545 (2008).

[14] B. M. Garraway, Phil. Trans. Royal Soc. A: Math.,Phys. and Eng. Sci. 369, 1137 (2011).

[15] J. Larson and M. Lewenstein, New Jour. Phys. 11 (2009).

[16] Y. Li, P. Zhang, and Z. D. Wang, Eur. Phys. J. D 58, 379 (2010).

[17] J. Keeling, M. J. Bhaseen, and B. D. Simons, Phys. Rev. Lett. 105, 043001 (2010).

[18] D. Nagy, G. Szirmai, and P. Domokos, Phys. Rev. A 84, 043637 (2011).

[19] S. Gopalakrishnan, B. L. Lev, and P. M. Goldbart, Nature Phys. 5, 845 (2009).

[20] S. Gopalakrishnan, B. L. Lev, and P. M. Goldbart, Phys. Rev. A 82, 043612 (2010).

[21] A. T. Black, H. W. Chan, and V. Vuletić, Phys. Rev. Lett. 91, 203001 (2003).

[22] A. F. Andreev and I. M. Lifshits, Sov. Phys. JETP 29, 1107 (1969).

[23] G. V. Chester, Phys. Rev. A 2, 256 (1970).

[24] A. J. Leggett, Phys. Rev. Lett. 25, 1543 (1970).

[25] G. Strang, SIAM J. Numer. Anal. 5, 506 (1968).

[26] S. A. Chin, Phys. Rev. E 76, 056708 (2007).

[27] V. I. Yukalov, Phys. Part. Nucl. 42, 460 (2011).

[28] P. G. De Gennes, Superconductivity of Metals and Alloys (Benjamin, New York, 1966). 
Corresponding author:

Ö. E. Müstecaplı̆̆lu

Office telephone number: +90212338 1424

Fax number: +90212338 1559

E-Mail address: omustecap@ku.edu.tr 